\title{
The Impact of Daily Kimchi Consumption: A Pilot Study
}

\author{
T Kraft, JF Keith*, B Bisha, E Larson-Meyer and A Griebel \\ Department of Family and Consumer Sciences, Laramie, USA
}

*Corresponding author: Jill F. Keith, Assistant Professor in the Department of Family and consumer Sciences, Laramie, WY, USA

\begin{abstract}
Background: Kimchi, a traditional fermented Korean food, contains prebiotics and probiotics, which have demonstrated ability to impact irregular gastrointestinal (GI) symptoms.

Objective: To evaluate the impact of daily kimchi consumption on GI symptoms, sensory characteristics and consumer acceptability of kimchi, and the microbial content in homemade and commercial kimchi.

Design: Dietary intervention study incorporating an experimental design. Participants consumed $75 \mathrm{~g}(1 / 2 \mathrm{cup})$ of kimchi twice a day for 14 days. Instruments included 3-day food records, a modified Gastrointestinal Symptom Rating Scale (GSRS), stool diaries using the Bristol Stool Scale (BSS), and a 7-point Hedonic scale (for sensory analysis and consumer acceptability). Lactic Acid Bacteria (LAB) were enumerated via plating methods.
\end{abstract}

Participants/Setting: Participants $(n=20)$ experiencing irregular GI symptoms were recruited from a Mountain West university community during fall 2017.

Main Outcome Measures: Main outcomes included GI symptoms, tracking of bowel movements, pre and post response to consumer acceptability, and LAB levels in homemade and commercial kimchi.

Analysis: Analysis included: 1) repeated measures ANOVA ( $p<0.05)$ for the GSRS and BSS with post-hoc testing for mean comparison between symptoms, 2) paired T-tests to assess mean differences in consumer acceptability and nutrient intake, and 3) culture-based microbial analysis with surface plating methods to determine bacterial counts.

Results: Participants reported a significant decrease in abdominal pain, heartburn, acid regurgitation, abdominal rumbling and distention, and eructation and gas production. Consumer acceptability and sensory characteristics questionnaires showed a majority of participants 'liked' kimchi and were willing to consume kimchi in the future. Bacterial analysis showed homemade kimchi had a lower concentration of LAB compared to commercial kimchi.

Conclusions: Kimchi is a nutrient dense probiotic source, acceptable among consumers in the study, with potential to impact irregular GI symptoms. Understanding consumer perception of kimchi provides valuable insight to when kimchi may be suggested as a method of probiotic and prebiotic intake.

Keywords: Kimchi; gastrointestinal; consumer acceptability; fermented foods; bacterial content; probiotics

\section{Introduction}

Fermentation has ancient roots in a variety of cultures. Kimchi, a fermented mixture of cabbage, salt, red peppers, radishes, and a variety of spices, has been a staple to Korean culture for over 4,000 years. [1,2] Lactic acid producing bacteria (LAB) produced during fermentation have been identified as factors that help maintain and improve gastrointestinal (GI) health. [3-5] Irregular GI symptoms affect $14.1 \%$ of the total U.S population. [6] The effects of irregular
GI symptoms are associated with a severe decrease in quality of life, and a substantial financial burden on society. [6] The dysbiosis of the microbiome and resulting irregular GI symptoms may be induced by a wide variety of circumstances that include consumption of a Westernized diet which is typically high in fat and refined carbohydrates and low in dietary fibre. [7] Supplementation of probiotics may mitigate dysbiosis of the microbiome. [8] Probiotics are live microorganisms which when administered in adequate 
amounts confer a health benefit on the host. [9] Research on the use of probiotics to alleviate dietary related GI disorders has shown potential. $[3,4,10]$ With current research demonstrating positive effects with supplementation of $\mathrm{LAB},[4,10]$ and the consumption of a variety of probiotics to maximize diversity and discourage the growth of harmful bacteria, [11] kimchi could provide a viable source of beneficial probiotics.

The bacteria promoted during kimchi fermentation have been demonstrated to act safely and effectively as probiotics. [12] Kimchi also contains prebiotics, such as inulin, a major food source for the growth of probiotic bacteria. Increased efficacy of probiotic supplementation has been demonstrated when prebiotics are present, lending credibility to claims that consumption of kimchi could positively affect the microbiome and improve irregular GI symptoms. [3,11] However, there is a paucity of literature addressing fermented foods in the Western diet and potential impact on the microbiome and GI symptoms. This pilot study examined 1) the impact of habitual consumption of kimchi on irregular GI symptoms and bowel form, 2) consumer acceptability and sensory characteristics of kimchi and 3) the microbial content in homemade and commercial kimchi.

\section{Methods}

During fall 2017, healthy participants ( $\mathrm{n}=20)$ aged $18-40$ years, experiencing irregular GI symptoms, but without history of malabsorptive or inflammatory bowel disease were recruited for a dietary intervention. Exclusion criteria included one or more of the following: diagnosed hypertension (due to high sodium content of kimchi), antibiotic use within past 3 months, current consumption of fermented products, excessive alcohol consumption (>3 drinks/day) (due to potential impact on GI microbiome) [13] and suppressed immune function (due to presence of live bacteria in kimchi). A sample size of 20 was estimated to provide an effect size of 0.8. [14] After initial phone screening to confirm eligibility, written, informed consent was obtained. The study was approved by the Institutional Review Board at the University of Wyoming. For this pilot intervention, participants consumed 75 grams ( $\sim 1 / 2$ cup) of kimchi twice a day for 14 days. Gastrointestinal symptoms were assessed at initiation, day 7, and day 14 using the Gastrointestinal Symptom Rating Scale (GSRS) [18] to evaluate the presence and severity of common symptoms associated with food intake.

The GSRS measures abdominal pain, heartburn, acid reflux, nausea and vomiting, borborygmus (stomach rumbling), abdominal distention, eructation (belching), gas production, decreased or increased passage of stools, hard or loose stools, urgent need to defecate, and satisfaction of bowel movement. The GSRS was modified from initial scale (0-3) to allow for consistency with other study questionnaires and each symptom was rated using a 7-point Likert Scale, where 1=no discomfort/presence of symptom and $7=$ severe discomfort/high frequency. Participants were asked to evaluate each bowel movement over the 14-days using the BSS (a common clinical tool used to evaluate GI transit time). [16] The BSS is a visual scale that depicts 7 types of common bowel forms, ranging from watery diarrhea with no solids (type 7) to constipation depicted as hard, separate lumps (type 1). Bowel formation of 1 or 2 was categorized as slow, 3 or 4 was normal, and 5-7 was fast.

The total number of slow, normal, and fast were represented as a percentage of total bowel movements during the week. Sensory characteristics and overall palatability were assessed by a 7-point Hedonic scale (7=extremely like, 1=extremely dislike) to rate appearance, flavour, texture, aroma, mouthfeel, and overall acceptability. Consumer acceptability and feasibility of including kimchi in their typical diet was assessed using a 7-point Likert scale ( 7 =strongly agree, 1 =strongly disagree) for five statements: 'I enjoy consuming fermented foods as part of my regular diet', 'I am aware of different types of fermented foods and how to prepare them', 'I am interested in learning more [5] about the different types of fermented foods', 'I am willing to eat kimchi in the future', and 'I plan on consuming kimchi regularly'. [17] Participants recorded a three-day food diary (including two weekdays and one weekend day) during each week of the study. Training on tracking dietary intake, portion sizes, and guidance to continue with regular diet patterns was provided by the lead researcher and a registered dietitian prior to the initiation of the study.

Food records were examined at the exit interview with discrepancies in portion sizes or unclear entries clarified to increase accuracy. Diaries were utilized to compare and contrast oral dietary intake during the study period and to promote adherence to the kimchi intervention and avoidance of other fermented foods. Threeday food records were analyzed with dietary analysis software (ESHA Food Processor Nutrition Analysis Software, ESHA Products, Salem, OR 2018).

\section{Bacterial Analysis}

Culture-based microbial analysis of the fermented kimchi was conducted to enumerate LAB. Three major genera are known to predominate within traditionally fermented kimchi; Leuconostoc, Lactobacillus, and Weissella. [18] Bacterial enumeration was conducted using surface plating on de Man, Rogosa, and Sharpe (MRS) agar (Thermo Fischer Scientific). X-gal (5Bromo-4-Chloro3-Indolyl $\beta$-D-Galactopyranoside) additive was purchased through Millipore Sigma, previously Sigma-Aldrich, St. Louis, MO. Ten grams of kimchi from the commercial product and homemade kimchi (prepared by a culinary-trained researcher in a controlled campus teaching kitchen) were resuspended in $100 \mathrm{ml}$ of sterile saline $(0.85 \%$ saline) solution in a WhirlPak® (Nasco, Fort Atkinson WI). The samples were mixed by hand for five minutes followed by serial dilutions using saline dilution blanks.

MRS agar or MRS agar supplemented with X-gal were utilized for surface plating. The supplementation with X-gal (chromogenic 
substrate for $\beta 6$ galactosidase) provided additional differential discrimination for Leuconstoc spp.19 Leuconostoc spp. are known to synthesize $\beta$-galactosidase enzyme, thus colonies precipitate a blue color upon plating. MRS is commonly used to isolate and enumerate LAB.20Incubation was performed inside an anaerobic chamber containing anaerobic sachets (Thermo Scientific Oxoid anaerogen $2.5 \mathrm{~L} \mathrm{Sachet)} \mathrm{at} 37^{\circ} \mathrm{C}$ for $72 \mathrm{hrs}$. Plating was performed in triplicates and counts were averaged to estimate kimchi microbial load as $\log 10 \mathrm{CFU} / \mathrm{g}$.

\section{Statistical Analysis}

Descriptive statistics were performed on all variables. Repeated measures ANOVA was used to assess significant changes in symptoms reported on the GSRS with post-hoc testing (Least Significant Difference, LSD) for mean comparison between symptoms. Frequency of slow, normal, and fast bowel movements reported on the BSS diaries were compared between the first and second week to identify improvement or changes in typical bowel formation and analyzed using repeated measures ANOVA. Posthoc testing using LSD determined differences between categories. Consumer acceptability pre- and post-intervention was analyzed using a paired T-test. Mean intake of key nutrients including macronutrients, dietary fibre, and sodium were compared from week 1 to week 2 of the study using paired T-test analysis. All statistics were performed using SPSS 24.0 (IBS SPSS Statistics for Windows, Version 24.0. Armonk, NY: IBM Corp 2016). The statistical significance was defined as $\mathrm{p}<0.05$ for all estimates.

\section{Results}

Table 1: Demographics of participants $(n=21)$ consuming kimchi at a Mountain West university.

\begin{tabular}{|c|c|}
\hline Characteristic & n (\%) \\
\hline \multicolumn{2}{|l|}{ Sex } \\
\hline Male & $9(42.8 \%)$ \\
\hline Female & $12(57.2 \%)$ \\
\hline \multicolumn{2}{|l|}{ Age (years) } \\
\hline $18-20$ & $2(9.5 \%)$ \\
\hline $21-30$ & $15(71.4 \%)$ \\
\hline $31-40$ & $4(19.1 \%)$ \\
\hline \multicolumn{2}{|l|}{ Ethnicity } \\
\hline Caucasian & $15(71.4 \%)$ \\
\hline Hispanic/Latino & $2(9.5 \%)$ \\
\hline Asian/Pacific Islander & $3(14.3 \%)$ \\
\hline Other & $1(4.8 \%)$ \\
\hline Anthropometrics & $\operatorname{Mean}( \pm \mathrm{SD})$ \\
\hline \multicolumn{2}{|l|}{ BMIa } \\
\hline Male & $27.23( \pm 5.18)$ \\
\hline Women & $27.41( \pm 6.23)$ \\
\hline
\end{tabular}

Table 2: Changes in gastrointestinal symptoms as reported on the Gastrointestinal Symptom Rating Scale by participants.

\begin{tabular}{|c|c|c|c|c|}
\hline \multicolumn{2}{|r|}{ Gastrointestinal Symptoms } & Day 1 Mean $( \pm$ SD) & Day 7 Mean $( \pm$ SD) & Day 14 Mean ( \pm SD) \\
\hline Category 1 & Abdominal Pain, Heart burn and Acid Regurgitation & $8.2( \pm 3.1)$ & $6.4( \pm 4.3)^{* *}$ & $5.2( \pm 2.9)^{* *}$ \\
\hline Category 2 & Nausea and Vomiting & $2.2( \pm 1.6)$ & $1.4( \pm 0.8)$ & $1.4( \pm 0.7)$ \\
\hline Category 3 & Abdominal rumbling and Distention & $7.8( \pm 2.7)$ & $6( \pm 2.8)^{* *}$ & $5.1( \pm 2.3) * *$ \\
\hline Category 4 & Eructation (belching) and Increased gas production & $7.6( \pm 2.8)$ & $6.5( \pm 2.7)$ & $5.2( \pm 1.9) * *$ \\
\hline Category 5 & $\begin{array}{l}\text { Decreased passage of stool, Presence of hard stool, and } \\
\text { Feeling of incomplete evacuation }\end{array}$ & $7.9( \pm 4.2)$ & $7.1( \pm 2.8)$ & $5.7( \pm 1.9)$ \\
\hline Category 6 & $\begin{array}{c}\text { Increased passage of stool, Presence of loose stool, and Urgent } \\
\text { need to defecate }\end{array}$ & $7.9( \pm 4.2)$ & $7.9( \pm 4.7)$ & $7.9( \pm 3.4)$ \\
\hline
\end{tabular}

**Denotes significant difference from Day $1(\mathrm{p}<0.001)$.

Forty individuals were screened with twenty-one individuals meeting inclusion criteria. One participant was lost to attrition on day 6 of the intervention. The GSRS and BSS were collected from individuals who completed the study. Consumer acceptability and sensory [7] characteristics included all twenty-one participants. Participant demographics are displayed in Table 1. Study compliance was assessed using food records or feedback at the exit interview. Gastrointestinal symptoms on the GSRS were grouped into six major categories (Table 2). Symptoms in categories 1, 3, and 4 were improved $(\mathrm{p}<0.01)$ with kimchi consumption. Normal distribution was confirmed upon analysis using repeated measures

ANOVA. Kimchi consumption had no measurable effect on typical stool form. The frequency of slow and normal bowel movements increased slightly, but not significantly ( $p=0.673$ ).

Table 3 contains a complete breakdown of sensory characteristics. More than half $(57.1 \%)$ of the study population 'liked' the kimchi, and an additional 9.5\% "extremely liked" it. Aroma and appearance had the highest occurrence of 'neither dislike nor like' with $28.6 \%$ reporting for each. Minor changes in consumer acceptability were seen over the two-week period. The only statement to demonstrate a significant increase was 'I enjoy consuming fermented foods as part of my regular diet' 
$(p=0.007)$. Comparison of average intake of calories, fat, protein, carbohydrates (CHO), dietary fibre, and sodium ( $\mathrm{Na}$ ) are presented from the analysis. No significant differences between week 1 and 2 in Table 4. Sodium data for one substantial outlier was excluded were detected for any of the selected nutrients.

Table 3: Sensory characteristics reported by participants sampling kimchi at study initiation.

\begin{tabular}{|c|c|c|c|c|c|c|}
\hline & 'Dislike' & $\begin{array}{c}\text { 'Somewhat } \\
\text { dislike' }\end{array}$ & $\begin{array}{l}\text { 'Neither dislike } \\
\text { nor like' }\end{array}$ & 'Somewhat like' & 'Like' & 'Extremely like' \\
\hline Appearance & $4.80 \%$ & $23.80 \%$ & $23.80 \%$ & $23.80 \%$ & $19 \%$ & $4.80 \%$ \\
\hline Aroma & $0 \%$ & $4.80 \%$ & $28.60 \%$ & $4.80 \%$ & $38.10 \%$ & $23.80 \%$ \\
\hline Flavour & $0 \%$ & $14.30 \%$ & $9.50 \%$ & $38.10 \%$ & $23.80 \%$ & $14.30 \%$ \\
\hline Mouthfeel & $4.80 \%$ & $0 \%$ & $23.80 \%$ & $14.30 \%$ & $33.30 \%$ & $23.80 \%$ \\
\hline Texture & $0 \%$ & $4.80 \%$ & $19.00 \%$ & $14.30 \%$ & $33.30 \%$ & $28.60 \%$ \\
\hline Overall & $0 \%$ & $0 \%$ & $14.30 \%$ & $19.00 \%$ & $57.10 \%$ & $9.50 \%$ \\
\hline
\end{tabular}

Table 4: Difference in average intake of selected nutrients between week 1 and week 2 as reported on 3-day food diary.

\begin{tabular}{|c|c|c|c|c|}
\hline Nutrient & Recommended intake & Week 1 Intake & Week 2 Intake & p-value \\
\hline Calories & Individualized & $2355 \pm 839$ & $2200 \pm 825$ & 0.382 \\
\hline Carbohydrate & $225-325 \mathrm{~g}$ & $255 \pm 112 \mathrm{~g}$ & $234 \pm 92 \mathrm{~g}$ & 0.302 \\
\hline Protein & $50-175 \mathrm{~g}$ & $88 \pm 25 \mathrm{~g}$ & $91 \pm 33 \mathrm{~g}$ & 0.261 \\
\hline Fat & $44-78 \mathrm{~g}$ & $112 \pm 54 \mathrm{~g}$ & $100 \pm 54 \mathrm{~g}$ & 0.359 \\
\hline Fibre & 25 g women 38 g men & $26 \pm 10 \mathrm{~g}$ & $24 \pm 9 \mathrm{~g}$ & 0.07 \\
\hline Sodium & $1,500 \mathrm{mg}$ & $4729 \pm 1567 \mathrm{mg}$ & $4100 \pm 1190 \mathrm{mg}$ & \\
\hline
\end{tabular}

**Denotes significant difference between week 1 and week $2(\mathrm{p}<0.05)$.

Consumption of $1 / 2$ cup kimchi twice a day and no additional fermented food intake was confirmed via the food diaries or verbally at the exit interview microbial counts of LAB were determined for both commercial and homemade kimchi. Commercial kimchi found LAB content when plated on MRS agar to be approximately 5.31 $\log \mathrm{CFU} / \mathrm{g}$. Commercial kimchi plated on MRS with the addition of X-gal found content to be approximately $5.26 \mathrm{log}$ CFU/g. Based on results obtained from plating on X-gal supplemented media, no detectable levels of Leuconostoc spp. were discernible. Homemade kimchi plated on MRS and MRS + X-gal, presented slightly lower concentrations of $4.21 \mathrm{log} \mathrm{CFU} / \mathrm{g}$ and $4.26 \mathrm{log} \mathrm{CFU} / \mathrm{g}$ respectively with no Leuconostoc spp. detected.

\section{Discussion}

An emerging number of clinical studies have evaluated the use of probiotics to improve GI disorder. The majority of these studies place emphasis on encapsulated probiotic supplements or probiotic enriched fermented milks/yogurts containing a mixture of LAB and Bifidobacterium spp. A number of these studies have demonstrated subjective improvement with regards to GI symptoms such as belching-abdominal fullness, bloating after meals, difficulty with defecation, and stomach gurgling. [3,21,22] Further research has demonstrated increased efficacy of probiotic supplementation when combined with prebiotic food sources such as inulin compared to probiotics alone. [3,23] Little research is available on the potential of fermented foods to act as beneficial probiotic sources.

Microbiological evaluation of kimchi has demonstrated the presence of a wide variety of LAB with the ability to act safely as probiotics. [12,24] However, limited information is [11] available for the use of kimchi to improve GI symptoms. Studies of probiotic supplementation generally emphasize high concentrations of bacteria and a wide variety of probiotic strains to maximize alleviation of symptoms. [11] In the current study, significant subjective improvement for abdominal pain, heart burn, acid regurgitation, abdominal rumbling and distention, and belching and gas production was observed, which is consistent with other studies. $[3,21,22]$ Stool form was not significantly affected which was corroborated with non-significant changes on participants' BSS. Previous studies have found that probiotic supplementation has little effect on typical stool forms. [4,22] Despite a recent rise in popularity of functional fermented foods, little is reported on consumer acceptability, sensory characteristics, and preference for kimchi. A previous study by Jang et al, evaluating the acceptability of kimchi presented similar findings to the current study in that there was an overall liking and perception of kimchi.25 Jang et al. found that in general, U.S. panelists gave overall liking scores of 6-7 on a 9-point hedonic scale. 
The author described this as the liking of kimchi to be 'slightly, to moderately pleasant.' It should be noted that the higher liking of kimchi could have been related to participant's preconceived notion about fermented foods and kimchi in general. It is plausible that some of the participants were attracted to the study due to the opportunity to incorporate kimchi into their regular diet. Food diaries provided insight about the typical diet of participants and allowed for comparison of key nutrients between each week. No significant changes in dietary intake of key nutrients was detected. Caloric intake is based on individual needs, so there are no set averages to assess participant caloric intake. According to the Institute of Medicine, Acceptable Macronutrient Distribution Range (AMDR), 20-35\% of kcals should come from fat. [26] For the average $2278 \mathrm{kcal}$ consumed by participants in this study, fat intake was $106 \mathrm{~g}$ which provided $41.8 \%$ of total kcal, [12] above AMDR recommendations.

The AMDR for protein is $10-35 \%$ of kcals. Participant protein intake was $90 \mathrm{~g}$ which provided $15.8 \%$ of total kcal, meeting the AMDR recommendation. Finally, the AMDR for CHO is $45-65 \%$ of kcals. Average CHO intake was $245 \mathrm{~g}$ which provided $43.0 \%$ of kcal, just under the minimum recommendation. Diet, particularly dietary fibre, is a major component that influences GI function and microbiota. [27] Current recommendations for dietary fibre per day are $38 \mathrm{~g}$ for men and $25 \mathrm{~g}$ for women under age 50.26 Participant dietary fibre intake ranged from $13 \mathrm{~g}$ to $45 \mathrm{~g}$ with an average intake of $25 \mathrm{~g}$. The mean dietary fibre intake of all individuals 2 years and older in the U.S. population is $16 \mathrm{~g}$ per day. [28] Kimchi contributed an additional $4 \mathrm{~g}$ of dietary fibre per day per participant which contributed to dietary fibre intake higher than the national average.

Sodium consumption among participants was well above the $1,500 \mathrm{mg} /$ day recommendation for both men and women, [29] with an average intake of $4,414 \mathrm{mg}$. It was also above the average daily sodium intake $33,400 \mathrm{mg}$ ) for individuals over 2 years of age. [30] Consumption of kimchi contributed to this high average intake, as it added 1,400 mg of sodium per day. Kimchi has been evaluated for the impact an individual's health. [24,3133] However, limited literature elucidates the potential for kimchi to act as a safe and effective probiotic source. $[12,24,34]$ In the current study, bacterial evaluation denoted that the commercial kimchi had LAB concentrations of approximately 5. [31] log $\mathrm{CFU} / \mathrm{g}$, which is lower than the suggested concentrations found within traditional fermented kimchi (8log CFU/g) [35] and what is described on the commercial product packing (8.16 log CFU/g). Bacterial concentrations in kimchi are influenced by a variety of factors, specifically variations in raw materials such as vegetable type, harvesting area, season, and supplemental ingredients. [18] Furthermore, LAB has been described as comprising 68.7-98.1\% of total bacteria present in kimchi. [35] This study was also not able to detect presence of 13 Leuconostoc spp. which are major contributors to the fermentation process.
The reasoning behind the lack of Leuconostoc spp. remains unclear. Leuconostoc spp. have been seen to predominate in the earlier stages of fermentation,18,36 thus microbial succession could be a reason. However, some strains such as, Le. gasicomitatum, have been seen to increase in later stages of fermentation, [37] which should be detectable by the plating methods utilized in our study due to their $\beta$-galactosidase activity.38 Despite the unclear reasoning for the lack of detectable Leuconostoc spp., this may prove to be advantageous for classifying kimchi as a probiotic rich food. Leuconostoc spp. have demonstrated low survival rates when passing through the GI tract31 which is vital for the classification as a probiotic. However, Lactococcus and Lactobacillus spp. have been validated to act as efficient probiotic bacteria. [24] While the microbial analysis followed previous literature for developing laboratory protocols $[18,19]$ the study was limited to analysing presence of LAB, not total microbial content, thus estimations cannot be extrapolated to all commercial products. More so, while this study demonstrated improved symptoms, participants were aware of the intent of the study which could have induced the placebo effect.

\section{Conclusions \& Recommendations}

Kimchi allows for inclusion of a nutrient dense vegetable source that has potential to impact GI health. Importantly, kimchi may provide a feasible and palatable method of supplementing probiotics within the diet and provides health professionals with an additional option to recommend to patients or clients. Further exploration of the impact of kimchi consumption would be beneficial in corroborating the evidence found within this study.

\section{References}

1. Jang DJ, Chung KR, Yang HJ, Kim K, Kwon DY et al. (2015) Discussion on the origin of kimchi, representative of Korean unique fermented vegetables. J Ethnic Foods 2(3): 126-136.

2. Ken-Yuon L, Hui YH, Ghazala S, Graham DM, Murrell KR et al. (2004) Fermentation: Principles and Microorganisms. eds. Handbook of Vegetable Preservation and Processing 1st ed. Monticello.

3. Basturk A, Artan R, Yilmaz A (2016) Efficacy of symbiotic, probiotic, and prebiotic treatments for irritable bowel syndrome in children: A randomized control trial. Turk J Gastroenterol 27(5): 439-443.

4. Kajander K, Myllyluoma E, Rajilic-Stojanovics M, Kyronpalo S, Jarvenpaa $S$ et al. (2007) Clinical trial: multispecies probiotic supplementation alleviates the symptoms of irritable bowel syndrome and stabilizes the intestinal microbiota. Aliment Phamacol Ther 27(1): 48-57.

5. Park J, Oh S, Cha Y (2014) Lactobacillus brevisOPK-3 isolated from kimchi inhibits adipogenesis and exerts anti-inflammation in 3T3-L1 adipocyte: L. brevisOPK-3 isolated from Kimchi inhibits adipogenesis. J Sci Food Agric 94(12): 2514-2520.

6. Hungin APS, Chang L, Locke GR, Dennis EH, Barghout V (2005) Irritable bowel syndrome in the United States: Prevalence, symptom patterns and impact. Aliment Pharmacol Ther 21(11): 1365-1375.

7. Tomasello G, Mazzola M, Leone A, Farina F, Cappello F et al. (2016) Nutrition, oxidative stress and intestinal dysbiosis: Influence of diet on gut microbiota in inflammatory bowel diseases. Biomed Pap Med Fac Univ Palcky Olomouc Czech Repub 160(4): 461-466. 
8. Conway PL (1996) Selection criteria for probiotic microorganisms. Asia Pac J Clin Nutr 5(1): 10-14.

9. Food and Agriculture Organization and World Health Organization Expert Consultation. Evaluation of health and nutritional properties of powder milk and live lactic acid bacteria. Córdoba, Argentina: Food and Agriculture Organization of the United Nations and World Health Organization; 2001.

10. Mezzasalma V, Manfrini E, Ferri E, La Ferla B, Nobile V et al. (2016) A Randomized, Double-Blind Placebo-Controlled Trial: The Efficacy of Multispecies Probiotic Supplementation in Alleviating Symptoms of Irritable Bowel Syndrome Associated with Constipation. Biomed Res Int.

11. Ng Chien S, Lam E, Lam T, Kamm MA, Chan Fk et al. (2013) Effect of probiotic bacteria on the intestinal microbiota in irritable bowel syndrome. J Gastroenterol Hepatol 28(10): 1624-1631.

12. Yosep J, Kim H, Park H, Lee H, Bong joon Kim et al. (2013) Functionality and safety of lactic bacterial strains from Korean kimchi. Food Control 31(2): 467-473.

13. Engen PA, Green SJ, Voigt RM, Forsyth CB, Keshavarzian A (2015) The gastrointestinal microbiome alcohol effects on the composition of the intestinal microbiota. Alcohol Res 27(2): 223-236.

14. Chang MS, Minaya MT, Cheng J, Connor BA, Lewis SK et al. (2011) Doubleblind randomized controlled trial of Rifaximin for persistent symptoms in patients with celiac disease. Dig Dis Sci 56(10): 2939-2946.

15. Kulich KR, Madisch A, Pacini F, Pique JM, Regula j et al. (2008) Reliability and validity of the Gastrointestinal Symptom Rating Scale (GSRS) and Quality of Life in Reflux and Dyspepsia (QOLRAD) 6(12).

16. Blake MR, Raker JM, Whelan K (2016) Validity and reliability of the Bristol Stool Form Scale in healthy adults and patients with diarrheapredominate irritable bowel syndrome. Aliment Pharmacol Ther 44(7): 693-703.

17. Megido RC, Geirts C, Blecker C, Chloe Gierts, Yves Broustaux et al. (2016) Consumer acceptance of insect-based alternative meat products in Western countries. Food Qual Prefer 52(C): 237-243.

18. Jung JY, Lee Sh, Kim JM, Park MS, Madsen EL et al. (2011) Metagenomic Analysis of kimchi, a traditional Korean fermented food. Appl Environ Microbiol 77(7): 2264-2274.

19. Mathor AG, Kihal M, Prevost H, Divies C. (1994) Selective enumeration of Leuconostoc on Vancomycin agar media. Int Dairy Journal 4(5) 459-469.

20. Obinna-Echem PC, Kuri V, Beal J (2014) Evaluation of the microbial community, acidity and proximate composition of akamu, a fermented maize food. J Sci Food Agric 94(2):331-340.

21. Waitzberg D, Logullo L, Bittencourt A, Shiroma Gm, Torrinhas RS et al. (2013) Effect of symbiotic in constipated adult women- A randomized, double-blind, placebo-controlled study of clinical response. Clinical Nutr 32(1): 27-33.

22. Guyonnet D, Woodcock A, Stefani B, Trevisan C, Hall C (2009) Fermented milk containing Bifidobacterium lactis DN-173 010 improved selfreported digestive comfort amongst a general population of adults. A randomized, open label controlled, pilot study. J Dig Dis 10(1): 61-70.

23. Lee J, Rheem S, Yun B, Bohyun yun, Jun lee S et al. (2013) Effects of probiotic yoghurt on symptoms and intestinal microbiota in patients with irritable bowel syndrome. Int J Dairy Tech 66(2): 243-255.
24. Lee NK, Han KJ, Son SH, Eom SJ, SK Lee et al. (2015) Multifunctional effect of probiotic Lactococcus Lactis KC24 isolated from kimchi. J Food Sci Technol 64(2): 1036-1041.

25. Jang SH, Kim MJ, Lim J, Hong JH (2016) Cross-cultural comparison of consumer acceptability of kimchi with different degree of fermentation. J Sens Stud 31(2): 124-134.

26. Institute of Medicine, Health and Medicine Division. Dietary Reference Intakes for energy, carbohydrate. fibre, fat, fatty acids, cholesterol, protein, and amino acids (2002/2005). http://nationalacademies. org/hmd/reports/2002/dietary-reference-intakes-for-energycarbohydrate-fiber-fat-fatty-acids-cholesterol-protein-and-aminoacids.aspx. Published September 5, 2002. Accessed November 1, 2018.

27. So D, Whelan K, Rossi M, Morrison M, Holtmann G et al. (2018) Dietary fiber intervention on gut microbiota composition in healthy adults: a systematic review and meta-analysis. Am J Clin Nutr 107:965-983.

28. Hoy MK, Goldman JD (2014) Fiber intake of the U.S. population: What we eat in America, NHANES 2009-2010. https://www.ars.usda.gov/ ARSUserFiles/80400530/pdf/DBrief/12_fiber_intake_0910.pdf Published September 2014. Accessed November 2, 2018.

29. Institute of Medicine, Health and Medicine Division. Dietary Reference Intakes: Water, potassium, sodium, chloride, and sulfate. http:// nationalacademies.org/hmd/reports/2004/dietary-referenceintakes-water-potassium-sodium-chloride-and-sulfate.aspx. Published February 11, 2004. Accessed November 2, 2018.

30. U.S. Department of Health and Human Services, U.S. Department of Agriculture. What We Eat in America. NHANES 2013-2014.

31. Kim EK, An SY, Lee MS, Kim TH, Han SJ et al. (2011) Fermented kimchi reduces body weight and improvers metabolic parameters in overweight and obese patients. Nutr Res 31(6): 436-443.

32. Choi H, Noh JS, Han JS, Kim HJ, Han ES et al. (20130 A fermented vegetable, improves serum lipid profiles in healthy young adults: Randomized clinical trial. J Med Food 16(3): 223-229.

33. Ki MR, Ghim SY, Hong IH, Park Jk, Jeong KS et al. (2010) In Vitro inhibition of Helicobacter pylori growth and of adherence of cagA-positive strains to gastric epithelial cells by Lactobacillus paraplantarum KNUC25 isolated from kimchi. J Med Food 13(3): 629-634.

34. Park KY, Jeong JK (2016) Kimchi (Korean Fermented Vegetables) as a Probiotic Food. In: Watson RR, Preedy VR, eds. Probiotics, Prebiotics, and Synbiotics: Bioactive Foods in Health Promotion. London, UK: Elsevier Inc.

35. Park EJ, Chun J, Cha CJ, Park WS, Jeon CO et al. (2012) Bacterial community analysis during fermentation of ten representative kinds of kimchi with barcoded pyrosequencing. Food Microbiol 30(1): 197-204.

36. Lee SH, Jung JY, Jeon CO (2015) Source tracking and succession of kimchi lactic acid bacteria during fermentation. J Food Sci 80(8): M1871M1877.

37. Jeong SH, Lee SH, Jung JY, Choi EJ, Jeon CO (2013) Microbial succession and metabolite changes during long-term storage of kimchi. J Food Sci 78(5): M763-M769.

38. Bjorkroth KJ, Geisen R, Schillinger U, Devos P, KorkealaHJ et al. (2000) Characterization of Leuconostoc gasicomitatum sp. nov., associated with spoiled raw tomato-marinated broiler meat strips packaged under modified-atmosphere conditions. Appl Environ Microbiol 66(9): 37643772. 
(C) (i) This work is licensed under Creative

To Submit Your Article Click Here: Submit Article

DOI:_10.32474/SJFN.2019.01.000120

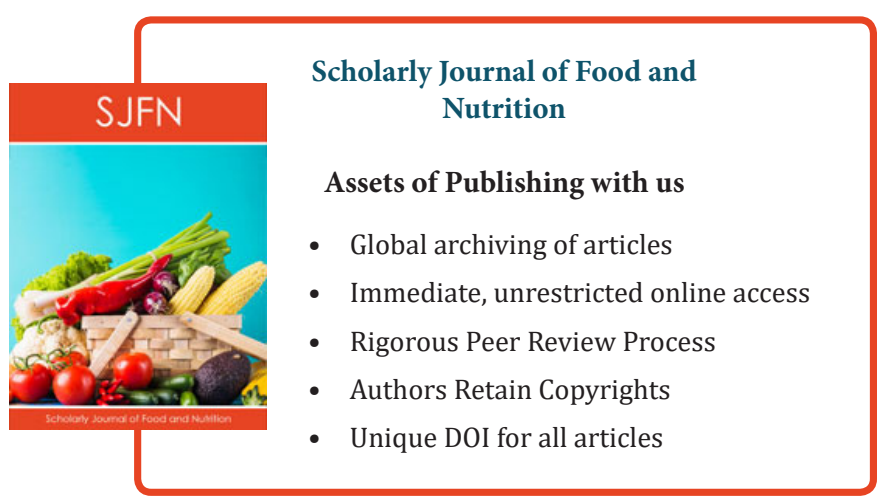

